\title{
Activation of Potassium Channels in Chick Skeletal Muscle by Extracellular ATP
}

\author{
RICHARD I. HUME ${ }^{a}$ AND STEVEN A. THOMAS
}

\author{
Department of Biology \\ Natural Science Building \\ University of Michigan \\ Ann Arbor, Michigan 48109
}

In developing chick skeletal muscle, micromolar concentrations of extracellular ATP elicit an early excitatory response followed by a late potassium conductance increase.' The potassium conductance activates with a delay of approximately one second and is greatly reduced at low temperature, suggesting that a second messenger may be involved. To examine the mechanism of activation of the potassium channels, we recorded from myoballs using the whole-cell and single-channel patch-clamp configurations.

When whole-cell recordings were made from myoballs held at the reversal potential for the early excitatory current, ATP elicited an outward potassium current. Noise analysis yielded spectral estimates for the single-channel conductance of approximately $20 \mathrm{pS}$. To determine whether these channels are activated by a second messenger, recordings were made from cell-attached patches while ATP was applied outside the pipette. It seemed unlikely that a highly charged molecule like ATP could cross the membrane-pipette seal, so we expected that channels within the patch should only be activated if this system used a second messenger. We observed the opening of potassium channels in response to ATP application outside of the pipette (FIG. 1) in about $60 \%$ of the cell-attached patches. To be sure that ATP was not gaining direct access to the pipette interior, some of these experiments were performed in the presence of the ATP receptor antagonist DIDS. When both DIDS and ATP were added to the solution outside of the pipette there was no activation of channels in the patch. However, when DIDS was present inside of the patch pipette, but not in the bathing solution (so as to selectively block the response to any ATP that managed to get across the seal), ATP applied outside of the pipette was still able to evoke potassium channel opening. These experiments indicated that the potassium channels must be activated by a second messenger.

The opening of potassium channels in response to ATP could also be observed in outside-out patches (FIG. 2). This result strongly suggests that the second messenger is restricted to the plasma membrane. In excised patch recordings the cytoplasmic face of the membrane is bathed by a huge volume of solution, and it seems unlikely that the concentration of a cytoplasmic second messenger could rise enough to elicit a response. A membranous second messenger would not be diluted by the solution inside the patch pipette.

${ }^{a}$ To whom correspondence should be addressed. 


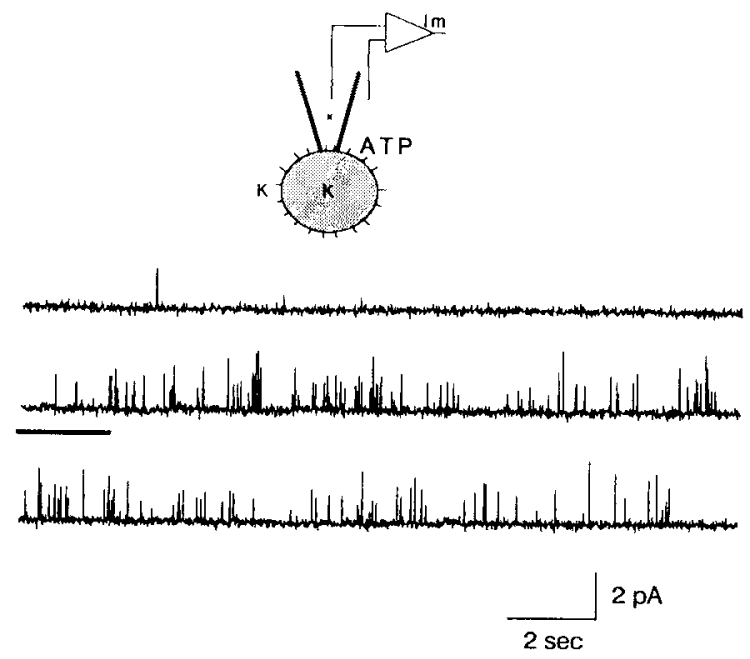

FIGURE 1. ATP activates potassium channels in chick skeletal muscle via a diffusible second messenger. Recordings were made from cell-attached patches in response to ATP applied outside of the pipette. The top trace was taken before ATP was applied. Very few potassium channels were active. ATP was applied during the middle trace (bar under trace). Many more potassium channels were active. The bottom trace was taken immediately after the middle trace. Once ATP was removed the potassium channel activity decreased.

FIGURE 2. The second messenger is probably restricted to the membrane. These traces show recordings from an outside-out patch in response to ATP. The top trace is the current across an outside-out patch before ATP was applied. ATP $(10 \mu \mathrm{M})$ was applied during the second sweep (bar under trace). The next four sweeps followed immediately after the application of ATP. Potassium channel openings in response to ATP are clearly seen. Because the volume of the solution bathing the inside of the patch is huge, it would have been expected that a cytoplasmic messenger would have been diluted too much to be active. The most likely explanation is that the second messenger remains within the membrane, and therefore is not diluted.
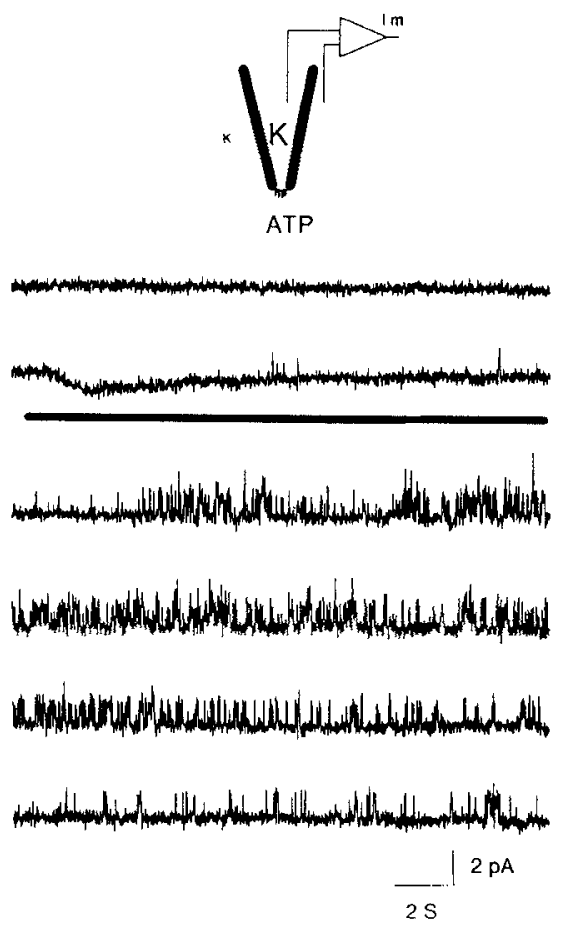
Pharmacological manipulation of myoballs indicated that activation of the potassium current did not depend on: 1) a rise in internal free calcium, 2) the activation of a $\mathrm{G}$ protein, or 3 ) protein phosphorylation. Taken together, these experiments indicate that the system that activates potassium currents in chick muscle is unlike any second messenger system that has yet been described.

\section{REFERENCE}

1. Hume, R. I. \& S. A. Thomas. 1988. Multiple actions of adenosine 5 -triphosphate on chick skeletal muscle. J. Physiol. 406: 503-524. 